\title{
Espacio, tiempo y necesidades cotidianas Herramientas de análisis y una mirada a la región Este de Uruguay
}

\author{
Space, Time and everyday needs \\ Analysis tools and a look to the Easter region of Uruguay
}

Nicolás Frank Gabin ${ }^{1}$

El artículo presenta avances de investigación referidos a la movilidad cotidiana de la población de la Región Este de Uruguay, que forman parte de la Tesis de Maestría (en curso). El objetivo principal del texto consiste en exponer las técnicas de análisis espacial desarrolladas por el autor en sucesivos trabajos, las innovaciones introducidas en la etapa actual, y los resultados primarios de su aplicación para la Región. A partir de información secundaria como la localización de servicios, vías de tránsito, y cursos de agua, se construyen áreas de influencia de los centros de servicios según seis niveles de necesidades. Esto permite identificar el área funcionalmente articulada -cubierta por servicios-, y la interrelación entre las distintas zonas. Se comienza por contextualizar las herramientas en los antecedentes teóricos y metodológicos, para luego desarrollar en profundidad los aspectos técnicos y su aplicación. Finalmente se presentan brevemente caminos de análisis que posibilitan los resultados.

Palabras clave: Geografía humana; SIG libre; Población rural; GRASS Gis

The article presents research advances related to the daily mobility of the population of the Eastern Region of Uruguay, which are part of the author's Master's Thesis (ongoing). The main objective of the text is to expose the spatial analysis techniques developed by the author in successive works, the innovations introduced in the current stage, and the primary results of their application for the Region. From secondary information such as the location of services, transit routes, and water courses, areas of

\footnotetext{
${ }^{1}$ Centro Universitario Regional Este - CURE, Universidad de la República, Uruguay.

Estudiante de la Maestría en Educación y Extensión Rural. Facultad de Veterinaria - UdelaR, Uruguay.

Dirección de Tesis: María Inés Moraes; Raquel Alvarado (co-directora).

nicolas.frank@cure.edu.uy
} 
2019| Tekoporá 1/ (1) Transformaciones territoriales en la región Este. Focus temático. Transformaciones...

influence of the service centers are built according to six levels of needs. This allows to identify the functionally articulated area -covered by services-, and the interrelation between the different zones. It begins by contextualizing the tools in the theoretical and methodological background, to then develop in depth the technical aspects and their application. Finally, analysis paths that the results enable are briefly presented.critically.

Keywords: Human Geography; Open Source GIS; Rural Population; GRASS Gis 


\section{INTRODUCCIÓN}

La propuesta de reflexionar sobre la dinámica sociodemográfica de una región como la comprendida por los departamentos del Este de Uruguay (Maldonado, Lavalleja, Rocha, Treinta y Tres y Cerro Largo) invita a colocar miradas y preguntas que transgredan los límites departamentales y municipales, y recuperen el movimiento real de necesidades y posibilidades de sus habitantes. En particular, la preocupación sobre el análisis de la movilidad cotidiana por trabajo, estudios y acceso a servicios incluida en la convocatoria del Coloquio requiere la consideración de las múltiples escalas en las que estas necesidades se manifiestan, en un conjunto articulado y diverso de territorios que van desde los espacios rurales hasta las áreas metropolitanas. El objetivo de este artículo es presentar en profundidad herramientas utilizadas para dar cuenta de estas dinámicas, así como resultados preliminares de su aplicación en los departamentos de la región Este.

Se presenta una técnica que parte del trabajo realizado en la Tesis de Grado en Geografia "Necesidades y posibilidades de la población rural. Una aproximación al conocimiento de su espacialidad" (Frank, 2012). Se trata de un conjunto de herramientas de análisis espacial que recuperan técnicas utilizadas para el estudio del 'Uruguay Rural' por el Centro Latinoamericano de Economía Humana (CLAEH, 1963), la Oficina Nacional de Acción Comunitaria y Regional del Ministerio de Trabajo y Seguridad Social (ACOR, 1990), el Instituto de Teoría y Urbanismo de Fac. de Arquitectura y el Programa de Construcción de la Demanda de MEVIR (Arq. Al ejandro Plada, com. pers.) entre otros. En diálogo con estos antecedentes se realizó una adaptación a las condiciones actuales del Uruguay y de las Tecnologías de Información Geográfica, realizando una reconceptualización en dos planos: técnico y teórico. En el primero, se adaptaron técnicas asociadas a la Teoría de la Localización de Instalaciones (facility location) y a la Geografía del Tiempo de Hägerstrand (Bosque \& Moreno, 2011) mediante el uso de Sistemas de Información Geográfica, para dar cuenta de las relaciones espaciales identificadas por los antecedentes mencionados. En el plano teórico, se recurrió a la conceptualización realizada por David Harvey (2006) sobre la espacio-temporalidad absoluta, relativa y relacional de los asuntos humanos.

Se obtuvo así "una técnica que permite saber, para cada punto de un territorio en particular sometido al análisis, su relación espacio-temporal con los servicios que dan satisfacción a las necesidades básicas de la población (...) de manera preliminar en base a información secundaria" (Frank, 2015:66). Esto implica por un lado, conocer para cada lugar cómo se da la articulación de los distintos niveles de servicios que van desde la escuela (urbana o rural) a la capital departamental, pudiendo reconstruir itinerarios potenciales en función de los distintos tipos de necesidad a satisfacer (saber qué lugares resulta esperable que se frecuentan desde donde y en qué tiempos). Por otro lado, permite también diferenciar un área funcionalmente articulada (Frank, 2012, 2015) -desde donde es posible acceder a los servicios presentes en los distintos centros mediante unas distancias-tiempo adecuadas a cada nivel de necesidad-, de un área funcionalmente no-articulada, la que -según estos criterios- no sería lugar apto para la reproducción social. En el mencionado trabajo la técnica fue aplicada en de departamento de Cerro Largo y validada mediante distintos métodos utilizando un volumen importante de información empírica.

Estas herramientas están siendo mejoradas y aplicadas en la investigación "El trabajo en la organización espacial del complejo agroindustrial arrocero de la Laguna Merín - Uruguay" correspondiente a la Tesis de Maestría en Educación y Extensión Rural (Facultad de Veterinaria, 
UdelaR). Allí, se pusieron en práctica para el análisis de los cinco departamentos mencionados en el comienzo, buscando identificar dinámicas de movilidad cotidiana a distintas escalas que contribuyeran como "Herramientas para el análisis de la acción colectiva de los trabajadores asalariados" del complejo arrocero.

En el comienzo del artículo se expondrán las innovaciones introducidas en las técnicas de análisis. Primero se hará una breve mención al plano teórico, para luego desarrollar las innovaciones que se están implementando actualmente en el plano técnico -automatizando los procesos en base a software libre-. Esto constituye el primer apartado (punto 2), en que se explican en detalle los procedimientos aplicados con el objetivo que puedan ser apropiados y/ o discutidos por otros investigadores. Luego, se presentarán los resultados preliminares del análisis espacial de la región, haciendo énfasis en dos aspectos. En primer lugar, la identificación provisoria del área funcionalmente articulada de la Región Este, lo que implica al mismo tiempo una cartografía de las importantes zonas desprovistas de todo servicio presentes en este territorio. Luego, se presentará también la articulación de las áreas de servicios a distintas escalas y niveles de complejidad, conformando mosaicos jerárquicamente articulados y espacialmente encajados que definen las áreas de influencia de los principales centros poblados respecto de los centros de menor complejidad y sus áreas de influencia rural correspondientes. Esto permite un análisis y discusión de sus configuraciones espaciales, que en muchos casos no respetan las jurisdicciones municipales ni departamentales.

\section{SOBRE LAS TÉCNICAS DE ANÁLISIS ESPACIAL UTILIZADAS}

Se mejoró y aplicó una técnica desarrollada en el marco de la Tesis de Grado en Geografia "Necesidades y posibilidades de la población rural. Una aproximación al conocimiento de su espacialidad" (Frank, 2012). Como se adelantara en la introducción, se trata de un conjunto de herramientas de análisis espacial que recuperan técnicas que comenzaron a utilizarse en la década del 60 del siglo XX para el estudio del 'Uruguay Rural' por parte de distintas instituciones y equipos de investigación (CLAEH, ACOR, entre otros). Estos antecedentes se basaban en los aportes de la economía espacial francesa de mediados del siglo XX, con ideas que "marcaron tanto el pensamiento espacial como la acción política" proponiendo un "desarrollo ordenado inducido por un ordenamiento consciente del medio de propagación” (Benko, 1999:88-89)

En diálogo con estos antecedentes se realizó una adaptación a las condiciones actuales del Uruguay y de las Tecnologías de Información Geográfica, realizando una reconceptualización en dos planos: técnico y teórico.

\section{RECONCEPTUALIZACIÓN TEÓRICA.}

En el plano teórico, resultaron útiles los aportes de Harvey (2006) sobre la espaciotemporalidad absoluta, relativa y relacional de los asuntos humanos, analizados en analogía con la teoría del valor de Karl Marx. Este enfoque permite analizar los diferentes marcos espacio-temporales implícitos en la acción humana en la sociedad capitalista, así como su interrelación. Así el autor conceptualiza mediante estos tres marcos relaciones espaciotemporales de diferente naturaleza que establecen los seres humanos: las relaciones de geometría fija, euclidiana, que explican la espacio-temporalidad absoluta de la fábrica, las mercancías, etc., asociadas al valor de uso y que podemos representar en términos de puntos líneas y polígonos. La espacio-temporalidad relativa del "mundo de movimiento" de la 
circulación de mercancías y realización del valor de cambio, que generan una geometría variable de acuerdo a los medios de transporte, velocidades y costos, no representables en el plano bidimensional -pero sí mediante la tecnología del SIG-. Y esto sin perder de vista la "tensión dialéctica" existente con el marco relacional que "internaliza la totalidad de la geografía histórica del proceso de trabajo, producción y realización de la mercadería, y la acumulación de capital en el espacio-tiempo del mercado mundial" (Harvey, 2006:xx).

Actuando el marco relacional como articulador del marco relativo y absoluto, es posible un análisis que tome en cuenta las relaciones espacio-temporales establecidas entre las personas y los servicios e infraestructuras producidos y fijados al suelo -analizados por los antecedentes vinculados a la economía espacial francesa-, y al mismo tiempo trascender los supuestos teóricos de su optimismo planificador, considerando así las distintas escalas involucradas en los acontecimientos locales y regionales a partir del análisis del movimiento del capital a escala global. En el trabajo de Frank (2015) puede verse un ejemplo de las posibilidades que permite la aplicación de este marco conceptual para el análisis de grupos sociales concretos, como es 'La dimensión espacio temporal de las estrategias colectivas de los trabajadores rurales asalariados y familiares'.

\section{AJUSTE Y APLICACIÓN DE LA TÉCNICA.}

En el plano técnico, para dar cuenta de las relaciones espaciales identificadas por los antecedentes teóricos anteriormente mencionados, se recurrió a una exploración y apropiación de las posibilidades abiertas por la tecnología de la información geográfica disponible en la actualidad. A continuación se detalla el procedimiento para la construcción de las herramientas de análisis, en base a los criterios definidos y validados en 2012 y su ajuste y mejora actualmente en curso en el marco de la Tesis de Maestría.

Primero se clasifican los centros de servicio según el tipo de necesidades humanas que permiten satisfacer, obteniendo seis clases nominadas de la $A$ a la $F$ de acuerdo a la complejidad de los servicios presentes en cada lugar. Se tomaron en consideración para la clasificación ocho tipos de servicios: Enseñanza; Salud; Cultural y esparcimiento; Industria y artesanía; Bancarios; Autoridad y administración; Personal y profesional. (CLAEH, 1963; ACOR, 1990; MEVIR, sd.; Frank, 2012, 2015).

Estas clases traen consigo una temporalidad asociada: El nivel $F$, el más simple, es el lugar requiere ser frecuentado en forma diaria, siendo la escuela primaria su servicio principal; El nivel A en cambio, los servicios allí presentes permiten un acceso más esporádico, siendo necesarios en forma semanal, mensual, etc. Por su parte, un centro de una determinada clase cumple su rol a ese nivel, pero también cumple el rol de todos los niveles inferiores. Tómese como ejemplo que, en la ciudad capital, nivel A, también hay escuelas primarias que se frecuentan diariamente en el nivel F.

Para aplicar la técnica en la presente investigación se partió de la clasificación realizada entre 2011 y 2013 por MEVIR (sd.) para todas las localidades de Uruguay reconocidas por el Instituto Nacional de Estadística (INE). Se obtuvo de MEVIR ${ }^{2}$ la base de datos correspondiente a la Región Este (154 centros poblados) la cual se georreferenció mediante el software QGis y se complementó con una base de datos de escuelas rurales y de servicios de salud obtenida de la geodatabase del Ministerio de Desarrollo Social. Se construyó así una base de datos espacial 
compuesta por 486 centros de servicio para los cinco departamentos que componen la región Este $^{3}$.

Partiendo del nivel más exigente en términos espacio-temporales se elaboró el área de influencia de todas las localidades en el nivel F a partir de la isocrona de media hora (Frank, 2012, 2015), construyendo para cada centro de servicios un área en el nivel F para la cual se espera que la población que allí reside acuda a ese centro por ese tipo de necesidad.

Esta tarea -que en 2012 se realizó en forma manual para Cerro Largo - se realizó en esta investigación de forma automatizada mediante el software GRASS Gis utilizando un modelo de datos ráster de resolución espacial $10 \mathrm{~m}$. Para ello se creó una capa de costos diferenciales (Bosque \& Moreno, 2011) mediante la herramienta r.mapcalc ${ }^{4}$ asignando velocidades a distintos tipos de vías de tránsito, ${ }^{5}$ al desplazamiento offroad y al cruce de cuerpos de agua que no ofician de barrera. ${ }^{6}$

Luego, en torno a cada centro de servicios se construyó mediante la herramienta r.cost la superficie de coste acumulado (ídem.) que indica para cada píxel el tiempo en minutos transcurrido desde el punto de origen, limitando el valor máximo a 30 minutos. Finalmente, para identificar las áreas de influencia de cada centro en el nivel $\mathrm{F}$ se tomó otro de los resultados de r.cost denominado 'basins', que adjudica un único valor a todos los píxeles cuyo trayecto más corto en distancia-tiempo corresponda a un mismo centro.

El Gráfico 1 es una captura de pantalla del software QGis donde se observa la superficie de coste acumulado creada mediante r.cost y representada en tonos de gris con sus respectivos puntos de partida. En la zona norte del área representada se observan en rojo el perímetro de los polígonos construidos manualmente por Frank (2012, 2015), apreciándose una coincidencia aceptable considerando que se utilizó en 2012 un dato de red de caminería anterior (menos actualizado, con una red más densa), y que existe variación en la localización de algunos centros de servicios (escuelas rurales).

En el Gráfico 2 se representan los polígonos resultantes del proceso de vectorización (mediante r.to.vect.area) de las áreas construidas con r.cost. Éstas corresponden con el área de influencia en el nivel $\mathrm{F}$ de todos los centros de servicio, y se representan como polígonos de

\footnotetext{
${ }^{3}$ En el transcurso de la investigación se pudo comprobar que la base de datos de escuelas -que coincide con la base oficial hoy disponible en la web de la Administración Nacional de Educación Públcia. Ver: https://sig.anep.edu.uy/siganep [Consultada 1 de Diciembre de 2018]- no es completa, actualizada ni en todos los casos correctamente georreferenciada. Varios de estos errores fueron subsanados para elaborar los resultados finales de la investigación que podrán encontrarse en la monografía final de la Maestría en Educación y Extensión Rural denominada: "El trabajo en la organización espacial del complejo agroindustrial arrocero de la Laguna Merín - Uruguay. Una aproximación al al análisis de la acción colectiva de los trabajadores asalariados." Realizada esta advertencia, se asume que los errores de la base de escuelas no invalida la discusión de los resultados preliminares que se presenta en este artículo. No obstante, en los gráficos y el mapa pueden advertirse algunas de las ausencias y errores señalados.

${ }^{4}$ Las herramientas de GRASS Gis suelen referirse mediante el nombre del módulo, lo que facilita su consulta en el Manual On-Line disponible en: https:// grass.osgeo.org/grass72/ manuals/**nombre_del_módulo**, o en la bibliografía especializada (ver Neteler y Mitasova, 2004). En este artículo cada vez que se menciona una herramienta de esta manera se refiera al software GRASS Gis.

${ }^{5}$ Red y categorización oficial elaborada por el Ministerio de Transporte y Obras Públicas, a las que se le asignaron velocidades.

${ }^{6}$ Los desplazamientos fuera de la red de caminos -comúnmente referidos en la bibliografía técnica como offroad- son fundamentales en la movilidad de la población rural a la escala de análisis necesaria para comprender las necesidades y posibilidades asociadas a la vida cotidiana. Para ello resulta importante considerar los cursos de agua, como barrera o como enlentecedores del desplazamiento, en vez de tomar el desplazamiento offroad como un espacio homogéneo. Esto fundamenta la necesidad de utilizar herramientas pertenecientes al modelo ráster de información geográfica, y no recurrir a opciones dentro del modelo vectorial como la extensión de GRASS visochrone que permite definir un único valor de velocidad offroad.
} 
borde verde y centro con relleno de trama de puntos. En la misma imagen se aprecian los centros de servicios analizados, las localidades INE con sus nombres y las vías de transporte y cuerpos de agua utilizados en la construcción de la superficie de coste diferencial. Por su relación con el problema de investigación para el cual fueron generados, se representan mediante polígonos de borde amarillo con relleno de líneas las áreas cultivadas con arroz en el verano de 2011, de acuerdo al dato elaborado por el Ministerio de Vivienda, Ordenamiento Territorial y Medio Ambiente mediante el método Land Cover Clasification System.

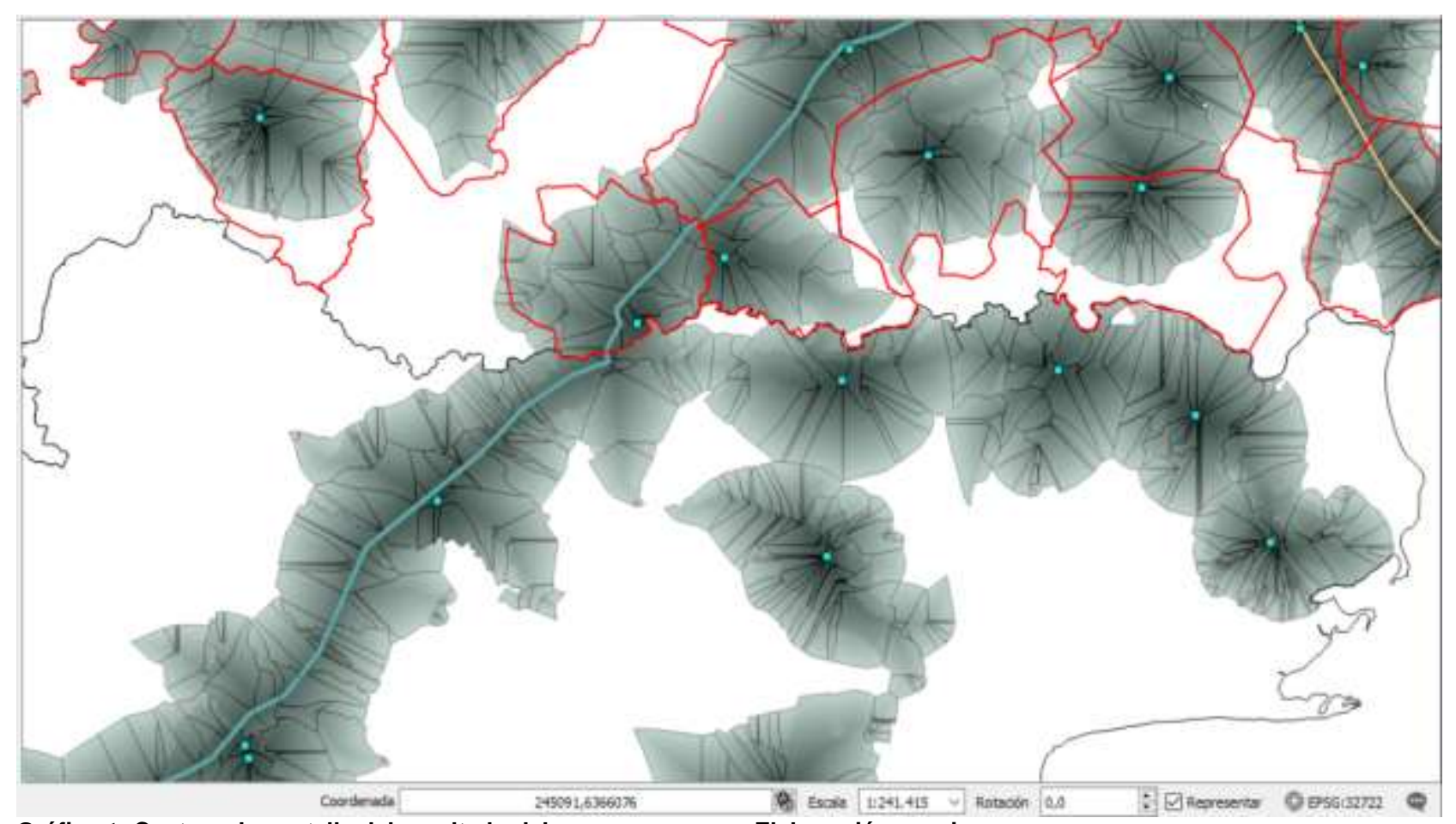

Gráfico 1: Captura de pantalla del resultado del proceso r. cost. Elaboración propia. 


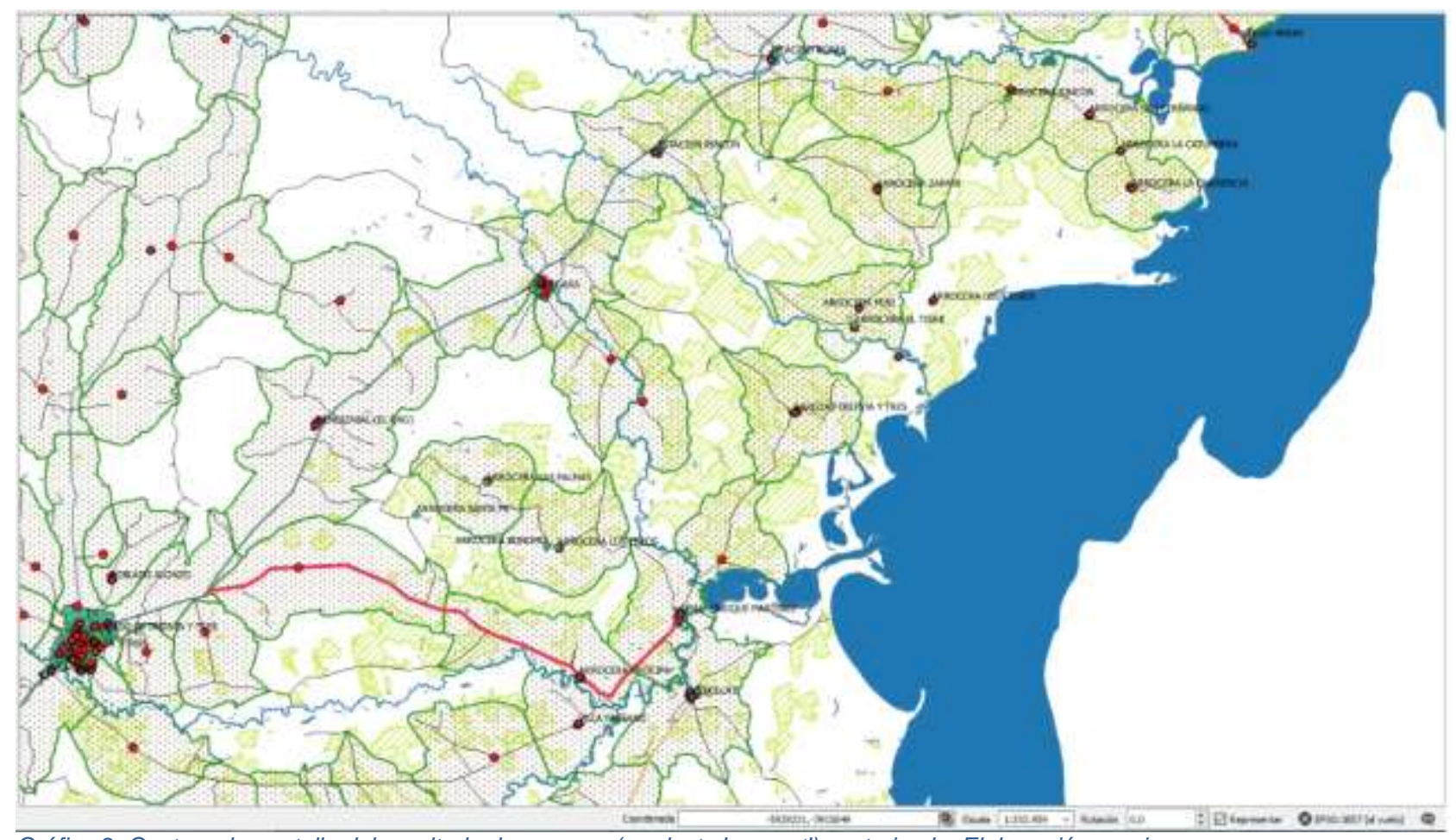

Gráfico 2: Captura de pantalla del resultado de r. cost (producto 'nearest') vectorizado. Elaboración propia.

Para construir las áreas de influencia en los otros niveles ( $A$ a E) se procedió mediante la agrupación de las áreas correspondientes a los centros de un nivel en torno a la localidad de nivel superior más próxima en distancia-tiempo (Frank, 2012, 2015). En la Figura 3 se observa como ejemplo la construcción de las áreas a nivel E. Para ello se tomaron las áreas de los centros de nivel $F$ y se asignaron a los centros de nivel $E$ o superior más próximos (representadas con círculos de color verde claro). Luego se filtraron y excluyeron todas las asociaciones que superaran la distancia-tiempo de una hora de centro a centro, dado que se considera que más allá de ese umbral no es razonable que la población pueda acceder con la frecuencia necesaria a los servicios del tipo $E$, principalmente servicios de salud del primer nivel de atención (Frank, 2012, 2015). 


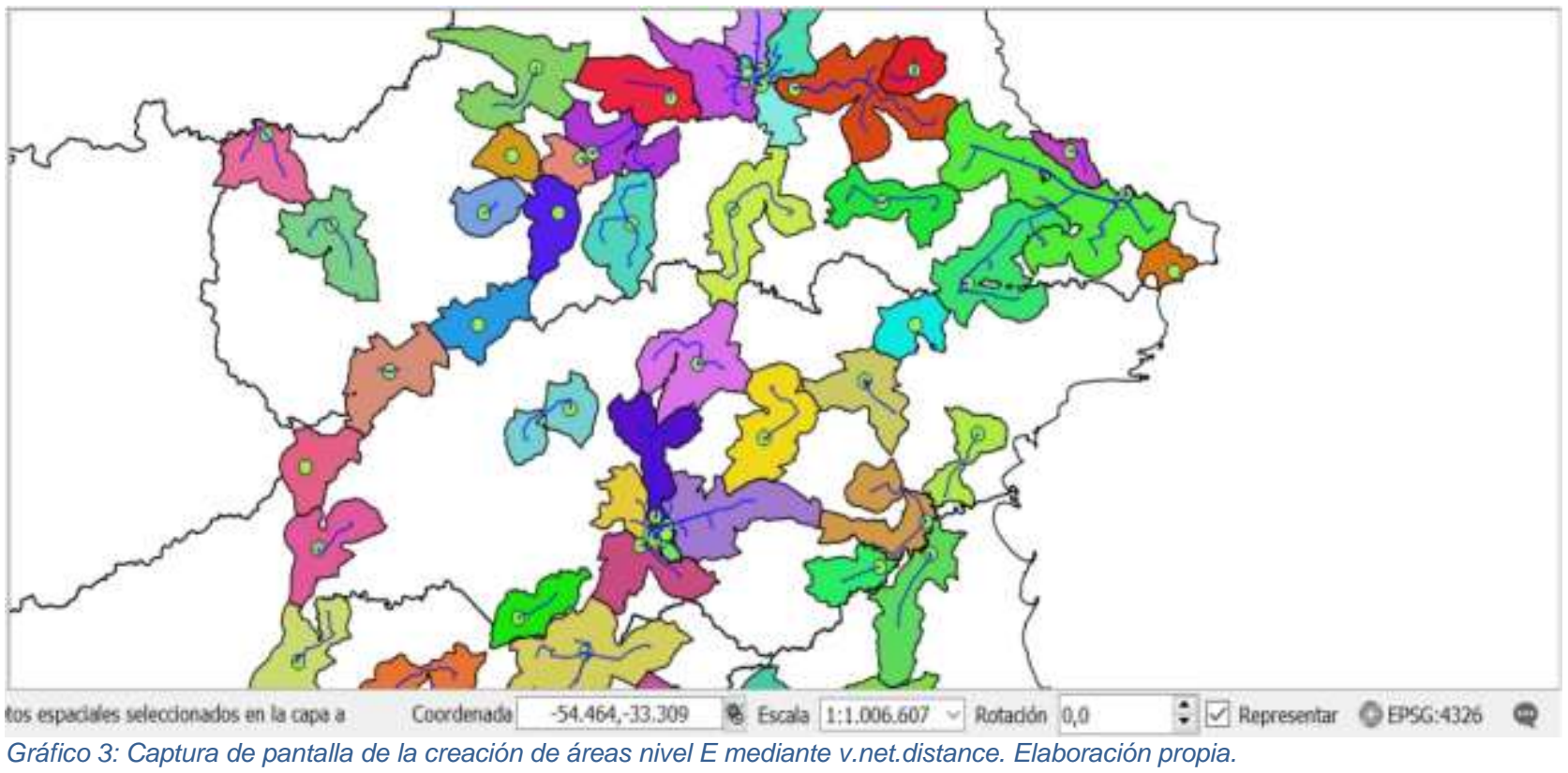

En esta investigación se realizó la asignación mediante un modelo de datos vectorial y análisis de redes, dado que no se consideró relevante la movilidad offroad para la evaluación de la distancia-tiempo de centro a centro. Se creó una red vectorial en base a la información oficial de vías de tránsito anteriormente mencionada, y se conectaron a la red los puntos de los centros de servicios mediante segmentos utilizando la herramienta v.net. Se le asignó a esos segmentos la misma velocidad que a la movilidad offroad utilizada anteriormente (10 km/ h), de modo que ambos modelos se comporten de manera equivalente. Para la conexión de puntos se estableció un umbral de 5km, descartando así 4 centros. Habiendo construido la base, y ajustado su topología para poder realizar los cálculos, se asignó cada centro de un nivel al centro más cercano de nivel superior mediante v.net.distance. En la Figura 3 se aprecian en azul los segmentos generados por la herramienta que corresponden al trayecto entre ambos centros mediante la red.

Procediendo por cada nivel hacia los centros de niveles superiores, se construyeron con el mismo procedimiento las seis áreas de influencia a los distintos niveles. Del nivel $D$ al $A$ no se estableció un umbral de distancia-tiempo, asumiendo que no existe impedimento para acceder al centro de servicios de niveles superiores dada la menor periodicidad con que se requiere el desplazamiento y la importancia de la necesidad que la motiva.

Este procedimiento asegura que el área de influencia de una localidad en su nivel de servicios correspondiente permita a todas las personas que habitan dentro de la misma a acceder a los servicios de los niveles iguales o inferiores a los de su cabecera a una distanciatiempo adecuada para cada uno. El área final en el nivel A incluye por tanto todas las áreas de menor complejidad y mayor demanda espacio-temporal y constituye el área funcionalmente articulada mencionada párrafos atrás. Su composición y posibilidades para la discusión se analizan en los apartados siguientes.

\section{RESULTADOS}

Como resultado de la adaptación y aplicación de la técnica se obtuvieron en esta etapa de la investigación siete productos interrelacionados. En primer lugar, una base de datos 
espacial compuesta por los 486 puntos correspondientes a cada centro de servicios y su respectiva clasificación en seis niveles. Luego, se obtuvieron los polígonos correspondientes para cada nivel. En el A son cinco objetos y en el otro extremo tenemos los 486 polígonos del nivel $\mathrm{F}$.

En el Mapa 1 se observa mediante colores las áreas en el nivel A, correspondiente a los únicos centros en ese nivel, las cinco capitales departamentales. Se representan también los centros en el nivel F, con la misma simbología que en la Figura 2. Como puede apreciarse, varias de estas áreas $F$ no forman parte de ningún área en nivel $A$, por no cumplir con los criterios de conectividad requeridos. Por lo tanto, solamente el área coloreada conforma el área funcionalmente articulada.

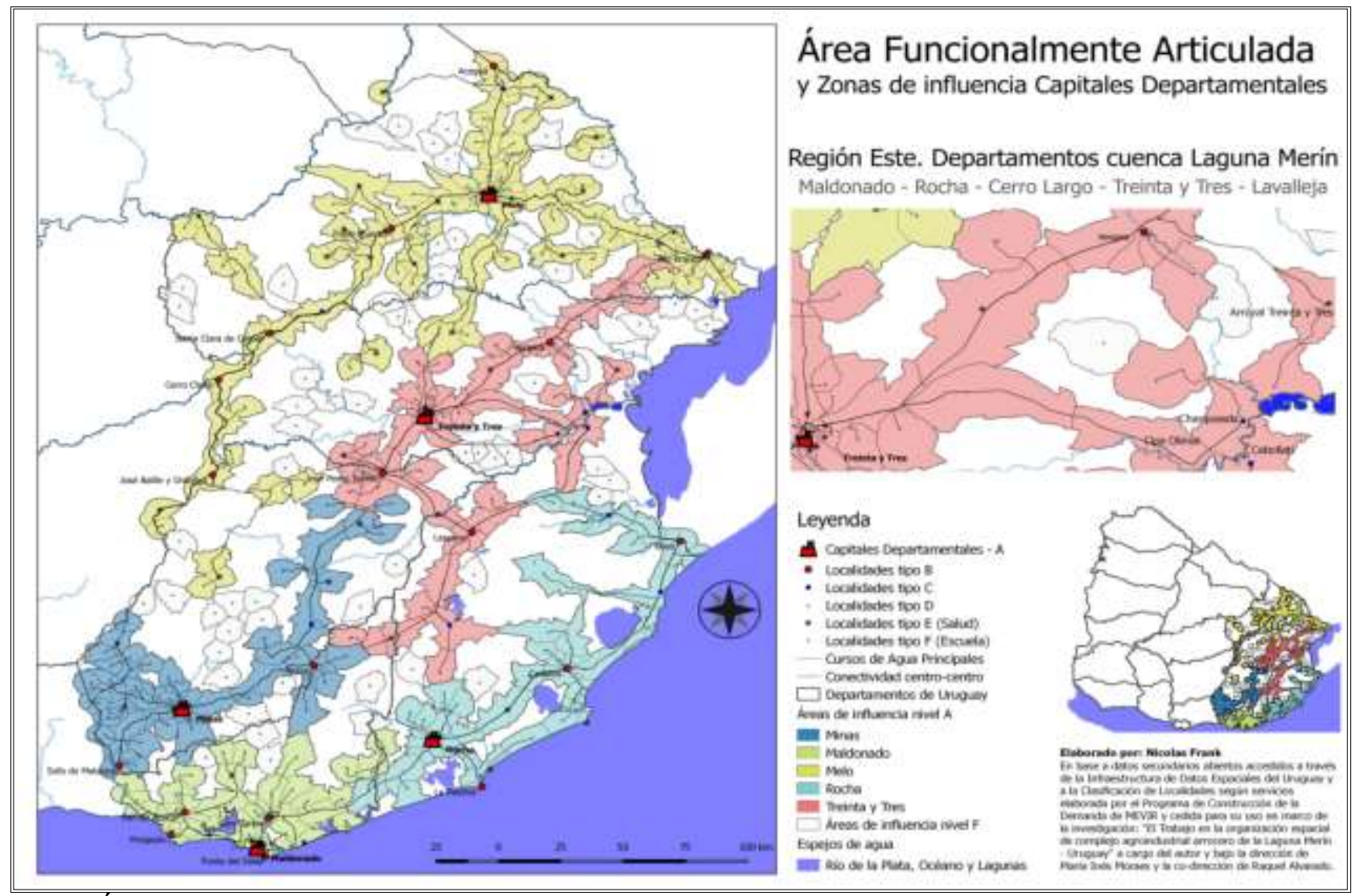

Mapa 1: Área funcionalmente articulada, áreas nivel A y áreas nivel F. Elaboración propia.

Como puede apreciarse, el área funcionalmente articulada corresponde a menos de la mitad de la superficie total de los cinco departamentos estudiados. Por lo que es extensa el área donde según los criterios empleados no sería posible la reproducción social de la población (rural) en los medios y condiciones actuales de la realidad uruguaya (Frank, 2012, 2015).

Por su parte, el uso combinado de los distintos niveles de servicios, permiten identificar para cada punto del mapa cuales son los centros de servicios más accesibles a la población para cada nivel de servicios. Como a cada nivel corresponde una temporalidad en particular, es posible identificar así con qué frecuencia y en qué lugares es esperable que interactúen las 
personas de distintas áreas de acuerdo a las necesidades y posibilidades de la vida cotidiana (Bertaux, 1983).

\section{DISCUSIÓN PRELIMINAR}

En primer lugar, cabe referirse al área funcionalmente articulada. Ya se mencionó su significado y extensión, por lo que cabe reflexionar sobre sus consecuencias concretas en la región Este, en lo referido a lo que implica su presencia-ausencia en los distintos territorios, análisis que en 2012 se denominaba dinámica externa. En ese trabajo, analizando caso a caso se identificó para Cerro Largo que "prácticamente todas las situaciones de zonas cubiertas por algún servicio y/o de pequeños predios, pero fuera del área funcionalmente articulada, así como todas las áreas de grandes extensiones cubiertas o no cubiertas (...), son situaciones problemáticas del punto de vista de las condiciones de vida y los procesos de diferenciación social que merecen atención particular" (idem.:58). Por su parte, se sugería que resultaba estratégica la posibilidad de articular el ejercicio teórico de construcción de las áreas - posible en base a información secundaria y que arroja resultados para toda la superficie estudidadacon el trabajo de campo, siendo la primera una vía para orientar la segunda.

En el contexto de investigación actual se aplicó la técnica sobre áreas 'conocidas' (zona arrocera de Laguna Merín y todo Cerro Largo) y otras zonas menos conocidas por el investigador. En las primeras, la herramienta aporta a identificar las zonas en que se produce arroz situadas fuera de los espacios donde es posible la reproducción social del trabajador y su familia (ej. Gráfico 2), permitiendo discutir las consecuencias que esto tiene para las actividades extralaborales como la organización sindical de quienes allí trabajan. Para las zonas menos conocidas, queda planteado como desafío el análisis de la situación de las extensas zonas comprendidas entre los principales ejes viales de la región, en particular entre las rutas 7 y 8 en Lavalleja y Treinta y Tres, así como entre la 8 y la 15 en la frontera de éstos con Rocha y Maldonado.

En cuanto a la dinámica interna, en el Mapa 1 se puede apreciar cómo las relaciones de articulación entre zonas no respetan los límites departamentales. ${ }^{7}$ En Frank (2012) se discutía el caso de Plácido Rosas (Dragón) en la frontera de Cerro Largo y Treinta y Tres por Ruta 18. Allí se advertía de la compleja situación jurisdiccional de la localidad por encontrarse más vinculada a Río Branco (sin pertenecer al Municipio) que a Melo, de quien dependía, ocasionando diversos inconvenientes a la población detectados en el trabajo de campo. Por su parte se advertía de su importante vínculo con Treinta y Tres, con problemas jurisdiccionales adicionales, pero no registrado en el modelo por estar fuera del área de estudio, a modo de 'efecto borde'. ${ }^{8}$ En el análisis actual fue posible evidenciar su relación con Treinta y Tres, debido al encadenamiento de relaciones intercentros, producto de analizar el centro de orden superior más cercano nivel a nivel, sin respetar los límites departamentales.

Nuevamente, el uso de la herramienta permite en este contexto de investigación orientar el análisis de las relaciones que la población del complejo arrocero establece con distintos centros en diversas espacialidades y temporalidades. En este caso enriqueciendo la identificación de espacios de convivencia para la población de distintas zonas que resultan

\footnotetext{
7

Ejemplos son Aiguá en Maldonado, José Pedro Varela en Lavalleja, Lascano y Cebollatí en Rocha, entre otros.

En los resultados aquí presentados debe utilizarse ese concepto de 'efecto borde' para relativizar el alcance del área de influencia de Melo por la Ruta 7, pudiendo estar influyendo otras localidades fuera del área de estudio. También al Sur sobre el Solís Grande.
} 
propicios para la interacción social. Queda planteado para futuros trabajos -y/o para el lector interesado- la discusión de las relaciones espaciales identificadas para otras zonas de la región Este.

\section{BIBLIOGRAFÍA}

Acor (1990) Rivera. Algunas características de centros poblados del departamento - Año 1990. Informe preliminar. Montevideo: Oficina Nacional de Acción Comunitaria y Regional - MTSS.

Benko, G. (1999) La ciencia regional. Bahía Blanca, Argentina: Universidad del Sur.

Bertaux, D. (1983) Sociología de la vida cotidiana y de relatos de la vida. Revista Suiza de Sociología. 9(1). (Traducción: Blanca Gabín).

Bosque Sendra J. \& Moreno Jiménez A. (Coords.) (2011) Sistemas de Información Geográfica y Localización Óptima de Instalaciones y Equipamientos. $2^{\circ}$ Edición. Madrid: Ra-Ma.

CLAEH (1963) Situación económica y social del Uruguay Rural. Montevideo: Ministerio de Ganadería y Agricultura.

Frank, N. (2012) Necesidades y posibilidades de la población rural. Una aproximación al conocimiento de su espacialidad. Tesis para obtener el título de Lic. en Geografía. Facultad de Ciencias. UdelaR.

Frank, N. (2015) La dimensión espacio temporal de las estrategias colectivas de los trabajadores rurales asalariados y familiares. Revista de Estudios Cooperativos. 19(1), 53-68.

Harvey, D. (2006) The Limits to Capital. London \& Brookling: Verso.

MEVIR (s.d.) Clasificación de centros según "Índice de Jerarquización Funcional IJF". Programa Construcción de la Demanda. Inédito.

NETELER, M. \& MITASOVA, H. (2004) Open Source GIS: A GRASS GIS approach. Second edition. Springer. 